\title{
On the claimed "circularity" of the theory of natural selection
}

\author{
Petter Portin \\ Laboratory of Genetics, Department of Biology, University of Turku, Turku, Finland \\ Email: petter.portin@utu.fi
}

Received 3 January 2012; revised 22 February 2012; accepted 19 March 2012

\begin{abstract}
First, the numerous claims that the theory of natural selection would be a tautology, just empty circular reasoning, are shown to be erroneous, and that they follow from an essentialistic and deterministic way of thinking, which is not consistent with the dynamic theory of evolution. Secondly, it is proposed that a careful analysis applying Fisher's Fundamental Theorem of Natural Selection of the seemingly tautologous sentence in question: "those who reproduce most, reproduce most" shows that in actual fact it is a predictive statement. Consequently, the analysis presented reduces the essence of the theory of natural selection to that one single statement.
\end{abstract}

Keywords: Evolution; Fitness; Fundamental Theorem; Tautology

\section{INTRODUCTION}

A formal argument against the theory of evolution, presented many times mainly by its opponents (e.g. [1]) but also by philosophers (e.g. [2,3]) and biologists [4], too, is that the theory of natural selection is circular. This argument follows from the definition of a central concept of the theory, the concept of "fitness".

Fitness is the relative capacity of an individual or genotype to produce fertile offspring, i.e. the average number of fertile progeny left by the genotype as compared to the average number of fertile progeny of other, competing genotypes [5]. Thus, the fittest are those individuals which reproduce most. On the other hand, those individuals which reproduce most are the fittest. From this a tautology follows: those who reproduce most, reproduce most.

The claim that the theory of natural selection would be tautologous is almost as old as the theory itself [3], and was presented by Bethell [1] approximately as follows [6]: Natural selection is defined by Spencer's phrase "survival of the fittest", but what does this term really mean? Who or what are the fittest? And how is "fitness" defined? Fitness involves no more than differential repro- ductive success, the production of more surviving offspring than other competing members of the population. But does not this formulation define fitness in terms of survival only? Based on this, the crucial phrase of natural selection would mean no more than "the survival of those who survive"-a vacuous tautology.

\section{DISCUSSION}

Hence, is the theory of natural selection a tautology, just empty circular reasoning? No, it is not.

As explained by Gould ([6], pp. 39-45; [7], pp. 368n369n), Charles Darwin himself, of course, had already presented several criteria of fitness which are independent of survival in his 1859 book "On the Origin of Species" [8]. These are found in its fourth chapter, entitled "Natural selection", and are summarized below.

Evolution is a response to changing environments. Thus, certain morphological, physiological, and behavioural traits should be superior a priori as designs for living in these new environments. Consequently, fitness can, in principle, be observed independently of survival. Criteria of fitness independent of survival can be applied to nature and have also been used consistently by evolutionists.

Or as Mayr ([9], p. 112n) explained, Darwin formulated a clearly non-tautological theory of natural selection by pointing out that in every generation there is a great overproduction of individuals, only a small percentage of whom can survive and reproduce. Secondly, all the individuals differ in their genetic endowment, and therefore differ, at least in principle, in their adaptedness to their common environment. And third, the causes of the differences in adaptedness are in part heritable. It follows by simple logic that those with the highest adaptedness have the greatest chance to survive and reproduce. In other words, adaptedness is a criterion of fitness independent of survival and reproduction.

What I seek to do here, is to show that the tautologous sentence in question becomes a predictive argument if it is realised that natural selection is a probabilistic and historical rather than a deterministic process. Consequently, 
it is still justified to define fitness as presented above in the second paragraph of this note. In fact, tautologies are always fine as definitions, but usually they cannot predict. It seems to me, however, that a tautology can be predictive if it contains comparative terms such as the word "most" in the present case.

Let us examine the content of the tautologous sentence presented above in which fitness is defined as the capacity to produce fertile offspring, namely the sentence "those who reproduce most, reproduce most”. This sentence necessarily implies that there are differences in the reproductive capacity, i.e. variance in the fitness of the individuals in a given population. According to Fisher's Fundamental Theorem of Natural Selection, first presented in 1930, "the rate of increase in fitness of any organism at any time is equal to its genetic variance in fitness at that time" ([10], p. 37).

From this theorem, it follows firstly that the evolution of a given species or population in general comes to a halt if the variation in fitness runs out: if there is no variation in fitness, there is no increase in fitness, and hence no evolution. Secondly, and more importantly, it follows that the average fitness of any population increases as long as there is genetic variation in fitness in the given population. This is the essence of the theory of evolution, and this conclusion also resolves the tautology presented above. Thus, the theory of natural selection is not circular reasoning. It is only seemingly tautologous.

\section{CONCLUSIONS}

Consequently, in actual fact, the apparently tautologous sentence presented, when carefully analysed, reduces the essence of the theory of natural selection to one single statement. Moreover, this tautology is actually a predictive argument which, when resolved, describes the course of biological evolution.

It seems to me that the claim presented by many philosophers of science that the theory of evolution is nonpredictive because of its circularity, and hence non-sci- entific (see [11]), follows from their essentialistic and deterministic way of thinking, which is not consistent with the dynamic theory of evolution. This view of mine I share with the great evolutionist Ernst Mayr ([9], p 112).

\section{ACKNOWLEDGEMENTS}

Thanks are given to Maaria Tringham, M.Sc., and Damon Tringham, M.Phil., for checking the language.

\section{REFERENCES}

[1] Bethell, T. (1976) Darwin’s mistake. Harper's Magazine, 75.

[2] Popper, K. (1974) Darwinism as a metaphysical research programme. In: Schillp, P.A., Ed., The Philosophy of Karl Popper, Open Court, LaSalle, 133-143.

[3] Rosenberg, A. (1985) The structure of biological science. Cambridge University Press, Cambridge, New York, Melbourne.

[4] Peters, R.H. (1976) Tautology in evolution and ecology. American Naturalist, 110, 1-12. doi:10.1086/283045

[5] Rieger, R., Michaelis, A. and Green, M.M. (1991) Glossary of genetics. Classical and molecular. 5th Edition, Springer Verlag, Berlin, Heidelberg, New York. doi:10.1007/978-3-642-75333-6

[6] Gould, S.J. (1977) Ever since Darwin reflections in natural history. Norton, New York, London.

[7] Gould, S.J. (2002) The structure of evolutionary theory. 5th Edition, Harvard University Press, Cambridge, London.

[8] Darwin, C. (1968) The origin of species. 6th Edition, Oxford University Press, London.

[9] Mayr, E. (1988) Toward a new philosophy of biology. Observations of an evolutionist. Harvard University Press, Cambridge, London.

[10] Fisher, R.A. (1958) The genetical theory of natural selection. 2nd Edition, Dover Publications, New York.

[11] Murray, B.G. Jr. (2001) Are ecological and evolutionary theories scientific? Biological Reviews, 76, 255-289. doi:10.1017/S146479310100567X 